\title{
Arterial tonometry in the classroom
}

\author{
Timothy Werner ${ }^{1}$ and Nabil Boutagy ${ }^{1}$ \\ ${ }^{1}$ Department of Health and Sport Sciences, Salisbury University, Salisbury, MD \\ ${ }^{2}$ Department of Human Nutrition, Foods, and Exercise, Virginia Tech, Blacksburg, VA \\ For correspondence: tjwerner@salisbury.edu
}

\begin{abstract}
The study of hemodynamic physiology utilizing traditional methods presents many challenges and limitations to educators. The goal of the educator is to provide the student with an adequate understanding of the basic hemodynamic anatomy and physiology governing blood flow, cardiac cycle and associated Korotkoff sounds, and the pathophysiology of vessel-related diseases. Several teachable moments reinforcing hemodynamic concepts are often overlooked when using traditional methods. The incorporation of arterial tonometry seeks to improve these shortcomings by offering a broader perspective of vascular physiology and introducing unique pathologies of the vasculature such as arterial stiffness and endothelial disorders.
\end{abstract}

Keywords: arterial tonometry, pulse wave velocity, pulse pressure, hemodynamics

\section{Introduction}

Arterial tonometry is a technique that involves measuring the speed at which pulses travel within the arterial system and the pressure changes they create. It allows the student to acquire and analyze important hemodynamic information not possibly obtained with brachial sphygmomanometry. A graph (see figures 1-2) of the pulse wave affords the user, in particular the student, the opportunity to assess pulse wave velocity (PWV), augmentation index (AI), central blood pressure, forward and reflective wave behavior, the dicrotic notch, central vs. peripheral pulse pressure differences, and timing intervals. By comparing graphs (Figure 3) of pulse waves from proximal and distal arterial sites, students can gain an appreciation of the amplification phenomenon and its clinical relevance. Students will also learn the physiological significance of arterial stiffness by simply comparing healthy and unhealthy pulse wave profiles (Figure 3).

In pathologic states, stiffening of arteries occurs via accumulation of collagen and cross linking), medial (smooth muscle) necrosis, calcification, and destruction of elastic lamellae. This results in the alteration of the viscoelastic properties of the arteries thereby increasing SBP and reducing DBP, hence increasing PP. In addition, an artery that cannot expand has no recoil. Therefore, DBP falls as the nature recoiling of elastic arteries is impaired. As a direct consequence of the stiffening process, PWV increases; similarly, the reflective wave travels back sooner. The reflective wave superimposes on the forward wave during systole in the aorta, augmenting left ventricular afterload and depleting coronary blood flow. A feed-forward system develops as the left ventricle compensates by hypertrophying, further increasing SBP and exacerbating stiffening of the arterial system. For a complete review, the reader is advised to these books and review articles (Laurent et al., 2006; McDonald, 1974; Milan et al., 2011; M. F. O'Rourke \& Hashimoto, 2008; Palatini et al., 2011; Salvi \& SpringerLink (Online service), 2012).

Arterial tonometry is an alternative and supplementary measurement tool to sphygmomanometry that the authors believe is imperative for curriculum in hemodynamic physiology. However, it is not without its critics (Cheng, Lang, Tufanaru, \& Pearson, 2013). The skill level to become proficient in arterial tonometry is similar to learning sphygmomanometry. Remind your students that patients and 
repetition are key virtues to learning. Students will gain a solid foundation in cardiovascular physiology and a better appreciation of the sophistication entailed within the human body.

\section{Arterial Tonometry}

Arterial tonometry is a technique that allows continuous and non-invasive measurement of arterial PWV and profile registration. In addition, it is less time consuming and more comfortable for the patient compared to other intra-aortic pressure procedures (i.e. cardiac catheterization).

Arterial tonometry requires the use of a high fidelity strain gauge pressure transducer placed on designated peripheral arterial sites, provided there is a hard structure underneath to support the artery. The radial artery is the most commonly used site due to its ease of palpation and support from the distal radius. However, many tonometry instruments have different programmed transfer functions, therefore making arterial tonometry necessary at alternative sites. These sites can include the common carotid artery supported by the larynx, brachial artery supported by the medial epicondyle, and femoral artery anchored between the pubic symphysis and iliac crest.

Regardless of artery site, DBP and mean arterial pressure (MAP) of the pressure wave must be obtained through sphygmomanometry at the site of the brachial artery (Mahieu et al., 2010). Systemic DBP and MAP show little to no variation throughout the arterial system during supine rest. Therefore, DBP and MAP in the large arteries is assumed to be the same as that obtained at the brachial artery. However, SBP increases along the length of the arterial tree in children and young adults, thus brachial SBP obtained from the brachial artery needs to first be integrated into the brachial waveform before obtaining SBP from the other arterial sites. Using a transfer function and proprietary algorithms, the tonometer unit computes SBP and other waveform parameters of the aorta, carotid, radial, and femoral arteries. There is some controversy in the literature over which artery to utilize as the calibration point when determining central blood pressure(Adji, O'Rourke, \& Namasivayam, 2011; Mitchell et al., 2010; M. F. O'Rourke \& Adji, 2012; M. F. O'Rourke, Safar, \& Roman, 2010). Regardless of this issue, the aforementioned brachial technique has been validated against, and correlated to more invasive measures(Laurent et al., 2006). Today, multiple indices and devices are available for measuring pulse waveforms. SphygmoCor ${ }^{\circledR}$, CardioMon ${ }^{\circledR}$, Pulsecor ${ }^{\circledR}$, Arteriograph ${ }^{\circledR}$, Complior ${ }^{\circledR}$, BPro ${ }^{\circledR}$, Vicorder ${ }^{\circledR}$, Mobil-O-Graph ${ }^{\circledR}$, Omron, and NIHem ${ }^{\circledR}$ are some of the more popular systems. Several models are very simplistic and cheap. Other models can involve complex algorithms, require trained operators and multiple sessions to complete, use different calibration methods, and cost thousands of dollars. Clearly, there is a trade-off with the practicability, accuracy, and reproducibility between the systems. The reader is directed to the following references of validation studies spanning several decades when comparing these factors and choosing a system most appropriate for educational purposes (Chen et al., 1996; De Melis et al., 2008; Gallagher, Adji, \& O'Rourke, 2004; Kelly \& Fitchett, 1992; Kracht et al., 2011; Naidu, Reddy, Yashmaina, Patnaik, \& Rani, 2005; Narimatsu, Takatani, \& Ohmori, 2001; Rogers et al., 2001; Vermeersch et al., 2008).

Additional information acquired by arterial tonometry centers around the pulse wave. The pulse wave is a wave created by left ventricular contraction that transmits along the walls of arteries. Much like the waves generated by the electrical event from the heart and captured with electrocardiograms, the contour, size, and speed of pulse waves can reveal cardiovascular abnormalities. Histological and molecular changes resulting in ridged arterial walls conduct faster pulse waves. PWV is a way of quantifying arterial stiffness by recording the speed of the wave, traditionally measured in $\mathrm{m} / \mathrm{s}$. Although blood flow velocity can provide interesting hemodynamic insights into ejection fraction, alveolar gas exchange, and several other pathologies, the velocity of blood flow does not influence PWV.

To provide a better understanding of PWV we recommend using visual aids to help explain the underlying physiological processes. Imagine a set of metal dominos standing equally dispersed, sideby-side in a long line across a quiet room. The first domino is struck and falls on to the domino beside it and the sequence repeats itself until the last domino is reached. With some exceptions, the arterial wall behaves much like a line of dominos and a few commonalities are worth noting. First, the length of the domino line does not change. The total length of the artery will not change as a pulse wave is 
traveling through it, though arteries will lengthen naturally as we age (M. O'Rourke, Farnsworth, \& O'Rourke, 2008). As a result, the longitudinal arterial expansion during each cardiac cycle is negligible, providing no assistance to blood flow. Second, the speed at which the dominos fall is independent of the speed of the first domino. PWV is unrelated to the strength of wave generation. Rather, it depends heavily on the collagen/elastin ratio of the aorta. Elastin fibers provide arteries (found principally in the aorta, common carotids, and subclavians) their characteristic distensible nature. Elastin allows the vessel to expand as blood is ejected through it during systole and recoil during diastole, thus pushing blood flow distally as the heart rests. Collagen fibers preserve the structural matrix of the arterial wall providing the ridged foundation necessary to resist high pressures. An artery with lower collagen/elastin ratios would conduct slower pulse waves because in a sense it is more distensible. Stiffer arteries conduct faster pulse wave velocity mainly due to the higher collagen/elastin ratio. Chronic exposure to oxidative stress, hyperglycemia, hypertension, and inflammatory compounds have all been shown to fracture elastin fibers while concomitantly creating more collagen crosslinking (and deposition) (Safar \& O'Rourke M, 2006). Lastly, all the dominos must fall before the wave reaches the end of the line. While probably the most obvious of all the analogies, the difference between the dominos falling and the wave created in the arterial tree is the generation of a reflective wave. This reflective wave will travel back to the heart with the same velocity as the forward wave. The arrival time of reflective waves in the aorta is one of the most significant hemodynamic concepts often overlooked in the physiology classroom and not attainable with sphygmomanometry.

A normal, healthy pulse wave contour has several peaks and valleys corresponding to the interaction between the forward and reflective wave (Figure 1). Importantly, the arterial site also affects wave contour, particularly its size, the further away the measurement is from the heart. There are several reasons for this, most notably the amplification phenomenon. Healthy arteries conduct slower pulse waves causing the forward and reflective to summate somewhere in the periphery. This is why PP is typically greater in the distal arteries of children and young adults (Figure 2).

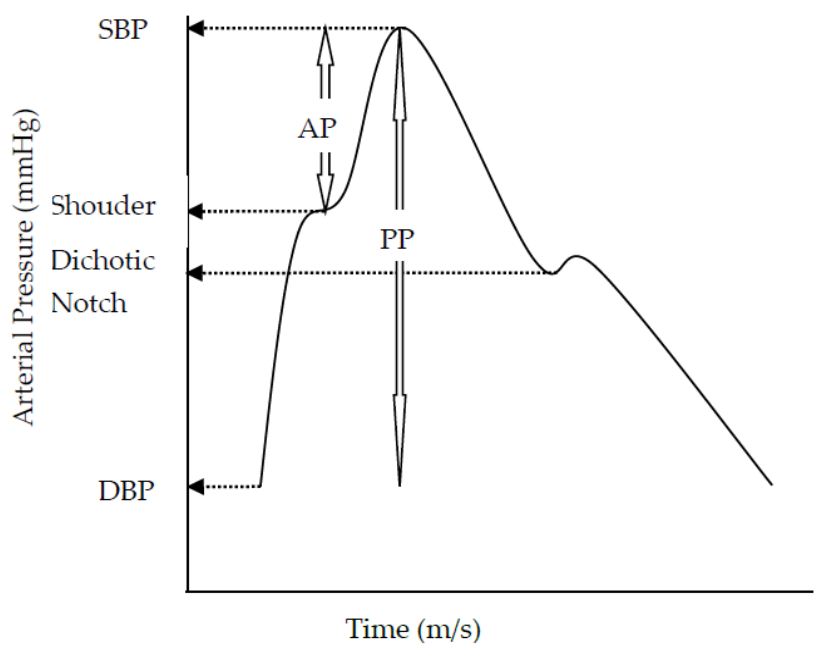

Figure 1. Cardiac Cycle 


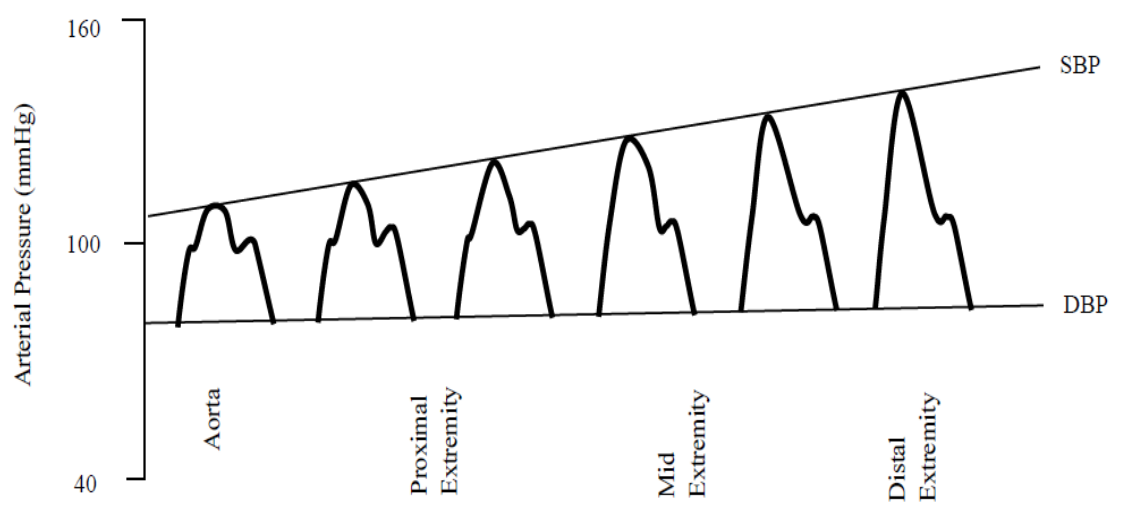

Figure 2. Changes in pulse wave contour of a healthy, elastic arterial system.

There are several markers on the pulse wave recordings corresponding to the pressure and timing of specific waves (Figure 3). Time is calculated by the simultaneous electrocardiograph ECG recording. The $\mathrm{R}$ wave serves as a fiducial point, indicating the initiation of pulse waves. The first markers correspond to the initial detection of the wave, often called 'Tfoot' and 'Pfoot' or time (ms) and pressure (mmHg) of the wave's beginning (foot), respectively. Naturally, there is a positive correlation between the timing of Tfoot and the arterial site's distance from the heart. Pfoot is the recorded diastolic pressure. It typically ranges from $70-80 \mathrm{mmHg}$ and should never measure zero as that would imply no arterial diastolic function. The second markers are labeled ' $\mathrm{Ti}^{\prime}$ and ' $\mathrm{Pi}^{\prime}$ indicating the arrival time and pressure of the reflective wave, respectively. In the literature this is sometimes known as the "shoulder". The shoulder can occur before or after the maximum wave or not at all. In central circulation, maximal waves followed by shoulders indicate early arrival of the reflective wave, augmenting the wave's peak. It is typical to see no shoulder in the brachial and radial waveforms because the impedance sites are closer to these arteries and the reflective wave's transit time is reduced. An individual with significant stiffness may also have carotid contours similar to brachial and radial contours as the stiffer arteries attenuate reflective wave transit time. Tmax and Pmax, the third markers, designate the timing and pressure of the wave's maximal amplitude, respectively. Tmax is usually the additive combination of the forward and reflective waves, though depending on the arterial site and degree of stiffness; this may not always be the case. Pmax represents systolic pressure and typically ranges from $110-130 \mathrm{mmHg}$. 'Tes' and 'Pes' are the last markers and record the timing and pressure at the end of systole, respectively and creation of the dicrotic notch. Often mistaken for the incisura, or the closing of the aortic semilunar valve, Tes and Pes indicate the recoiling nature of elastic arteries, principally of the aorta, further propelling blood flow distally. The amplitude of Pes declines as the aorta undergoes more collagen deposition and elastin fragmentation.

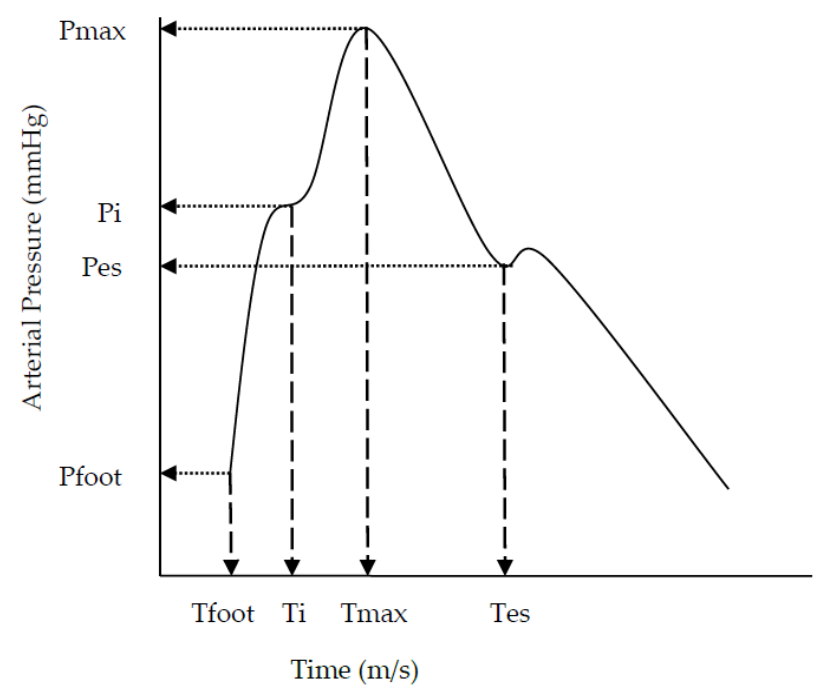

Figure 3. Pulse Wave 
Many of these markers are used in the calculations of clinically significant hemodynamic variables such as PWV (discussed previously), augmentation index (AI), (PP), and MAP. AI is the additional influence of the reflective wave on central maximal pressure and afterload. Reflective waves arriving during systole will have a positive impact on AI and can be one of many surrogates for arterial stiffness. Generally, higher positive AI values are associated with greater cardiovascular risk (Ageenkova \& Purygina, 2011; Chirinos et al., 2005; Crilly, Coch, Clark, Bruce, \& Williams, 2007). AI is the ratio of augmented pressure (AP) to PP. AP, or the absolute pressure increase due to the returning reflective wave, is calculated by subtracting carotid Pi from Pmax. An individual's height is negatively correlated to AI as longer arteries slow down transit time. Heart rate also demonstrates a negative relationship to AI. Slower heart rates tend to increase systolic ejection time, allowing the reflective wave to return during systole and augment maximal pressure. Because both healthy and unhealthy individuals can have higher absolute AI values, by convention, AI is positive when Pi occurs before Pmax and negative when Pi occurs after Pmax.

Subtracting Pfoot from Pmax provides an estimate of PP. In healthy individuals, PP increases distally as Pmax rises from the aorta to the periphery because of the amplification phenomenon. Stiffer arteries do not experience this, as there is compositional similarities and impedance matching between the central and peripheral arteries. The amplification phenomenon can also be calculated by subtracting carotid Pmax from brachial Pmax.

MAP is simply not the mean of systolic and diastolic pressure. Rather, because diastole composes roughly two-thirds of the cardiac cycle, greater emphasis is placed on diastolic pressures in the formula. It is calculated by adding $33 \%$ of PP to Pfoot. It should not significantly change throughout the arterial system while resting in a supine position.

\section{Implementation in the Classroom}

In order to establish consistency in arterial tonometry measurements, a universal consensus document was created to improve validity and repeatability between research groups and health care practices. Recommendations for student preparation and set-up are summarized here and discussed in greater detail elsewhere (Laurent et al., 2006). Students should refrain from consuming food and beverages (particularly containing caffeine) and tobacco based products at least three hours prior to testing. They should be resting comfortably in a supine position for at least 10 minutes in a climate-controlled room. During supine rest, the instructor should place the four limb lead electrodes in their corresponding areas and a blood pressure cuff over the right brachial artery of the student. By convention, all tonometry measurements are taken on the right side of the body. These are basic standards to avoid confounding influences.

The tonometry session begins by acquiring multiple blood pressures measurements until stabilization is reached. Ideally, three measurements are taken at least 2 minutes apart. Both the SBP and DBP must be within $+/-5 \mathrm{mmHg}$. If not, two more measurements are taken. The mean of the three sequential readings in the analysis are used to calibrate PP in the aorta, carotid, radial, and femoral arteries. Most modern tonometry units house an automated system and are preferred over manual measurements to reduce inter/intra-operator variability.

Arterial tonometry is taught alongside arterial pressure auscultation and electrocardiography within the cardiovascular curriculum. Because tonometry requires the use and skill of sphygmomanometry and electrophysiology (ECG), we recommend teaching these concepts first. Though a thorough understanding of electrophysiology is nonessential, students need a rudimentary understanding of lead placement and ECG waves before using the tonometer.

For all intents and purposes, the R wave is the only obligatory ECG wave while performing arterial tonometry. It serves as the fiducial or focal point indicating the approximate time of ventricular contraction. There is a slight delay, called the latent period, between the electrical event and mechanical event in cardiac muscle. However, this delay is consistent between cardiac cycles and will 
not affect the repeatability measurements within the same student. Effort should be made to avoid external artifact while obtaining data. The ECG should be stable, with tall, upright $\mathrm{R}$ waves.

As with most experiments, an introduction and demonstration of tonometry by the instructor helps to ease the tension and confusion when dealing with novel technology. Proper handling of the tonometer should be emphasized during this demonstration. It is extremely sensitive to pressure, not to mention expensive and special care must be give when handling it. Because the tonometer comes in direct contact with skin, cleaning it with alcohol swabs between students is ideal.

Proper technique is crucial when handling the tonometer. It is best to rest the fingers over the tonometer to minimize any tremors and baseline drift on the measurement. Begin by placing the tonometer directly over the strongest pulsation. Due to differences in the muscular and adiposity distribution between sites, special attention should be given among the four sites. Typically, the radial artery is the least challenging, whereas acquisition of the femoral and brachial can be problematic. Pushing down harder on the tonometer will not improve the image. The baseline pressure will wander if hold down pressure varies during the measurement. Patience and stable hands cannot be emphasized enough during the measurement. On average, proficiency is achieved after completing 100 measurements. While this is not realistic in a laboratory setting, students can appreciate the skill involved.

We recommend providing an assignment utilizing the student's individual pulse waves to enhance comprehension of the hemodynamic variables just learned. It should include calculations and questions on PP, MAP, PWV, AI, and amplification phenomenon. Application of the material can be demonstrated by comparing pulse waves between genders, physically trained vs. untrained, young vs. old, and stiff vs. unstiff arteries. Advanced classes should incorporate clinical situations involving pulse wave analysis as it relates to cardiovascular risk prediction. This will help capture some of the basic cardiovascular principles governing human physiology.

\section{Conclusions}

Arterial tonometry provides the student with novel learning opportunities and experience with advanced technology. Due to the limited teachable moments from sphygmomanometery, many of the fundamental cardiovascular concepts and hemodynamic physiological principles are often missed or overlooked. Teaching pulse waveforms will improve understanding of cardiovascular physiology, providing a bigger picture of overarching themes governing normal and pathological functions.

The importance of understanding arterial stiffness and the consequences associated with it cannot be understated. Pulse wave analysis provides valuable insight into the etiology and pathophysiology of arterial stiffness. The ability to measure and examine pulse wave characteristics will be vital in the future of medicine.

\section{References}

Adji, A., O'Rourke, M. F., \& Namasivayam, M. (2011). Arterial stiffness, its assessment, prognostic value, and implications for treatment. Am J Hypertens, 24(1), 5-17. doi: ajh2010192 [pii]10.1038/ajh.2010.192

Ageenkova, O. A., \& Purygina, M. A. (2011). Central aortic blood pressure, augmentation index, and reflected wave transit time: reproducibility and repeatability of data obtained by oscillometry. Vasc Health Risk Manag, 7, 649-656. doi: 10.2147/VHRM.S24877

Chen, C. H., Ting, C. T., Nussbacher, A., Nevo, E., Kass, D. A., Pak, P., . . Yin, F. C. (1996). Validation of carotid artery tonometry as a means of estimating augmentation index of ascending aortic pressure. Hypertension, 27(2), 168-175.

Cheng, H.-M., Lang, D., Tufanaru, C., \& Pearson, A. (2013). Measurement accuracy of non-invasively obtained central blood pressure by applanation tonometry: A systematic review and meta-analysis. International Journal of Cardiology, 167(5), 1867-1876. doi: http://dx.doi.org/10.1016/j.ijcard.2012.04.155

Chirinos, J. A., Zambrano, J. P., Chakko, S., Veerani, A., Schob, A., Willens, H. J., . . Mendez, A. J. (2005). Aortic pressure augmentation predicts adverse cardiovascular events in patients with established coronary artery disease. Hypertension, 45(5), 980-985. doi: 10.1161/01.HYP.0000165025.16381.44

Crilly, M., Coch, C., Clark, H., Bruce, M., \& Williams, D. (2007). Repeatability of the measurement of augmentation index in the clinical assessment of arterial stiffness using radial applanation tonometry. Scand J Clin Lab Invest, 67(4), 413-422. doi: 779335895 [pii]10.1080/00365510601131946 
De Melis, M., Morbiducci, U., Scalise, L., Tomasini, E. P., Delbeke, D., Baets, R., . . Segers, P. (2008). A noncontact approach for the evaluation of large artery stiffness: a preliminary study. Am J Hypertens, 21(12), 1280-1283. doi: 10.1038/ajh.2008.280

Gallagher, D., Adji, A., \& O'Rourke, M. F. (2004). Validation of the transfer function technique for generating central from peripheral upper limb pressure waveform. Am J Hypertens, 17(11 Pt 1), 1059-1067. doi: 10.1016/j.amjhyper.2004.05.027

Kelly, R., \& Fitchett, D. (1992). Noninvasive determination of aortic input impedance and external left ventricular power output: a validation and repeatability study of a new technique. J Am Coll Cardiol, 20(4), 952-963.

Kracht, D., Shroff, R., Baig, S., Doyon, A., Jacobi, C., Zeller, R., . . Melk, A. (2011). Validating a new oscillometric device for aortic pulse wave velocity measurements in children and adolescents. Am J Hypertens, 24(12), 1294-1299. doi: 10.1038/ajh.2011.147

Laurent, S., Cockcroft, J., Van Bortel, L., Boutouyrie, P., Giannattasio, C., Hayoz, D., . . Struijker-Boudier, H. (2006). Expert consensus document on arterial stiffness: methodological issues and clinical applications. Eur Heart J, 27(21), 2588-2605. doi: 10.1093/eurheartj/ehl254

Mahieu, D., Kips, J., Rietzschel, E. R., De Buyzere, M. L., Verbeke, F., Gillebert, T. C., . . Segers, P. (2010). Noninvasive assessment of central and peripheral arterial pressure (waveforms): implications of calibration methods. J Hypertens, 28(2), 300-305. doi: 10.1097/HJH.0b013e3283340a1a

McDonald, D. A. (1974). Blood flow in arteries (2nd ed.). London: Edward Arnold.

Milan, A., Tosello, F., Fabbri, A., Vairo, A., Leone, D., Chiarlo, M., . . Veglio, F. (2011). Arterial stiffness: from physiology to clinical implications. High Blood Press Cardiovasc Prev, 18(1), 1-12. doi: 10.2165/11588020-000000000-00000

Mitchell, G. F., Hwang, S. J., Vasan, R. S., Larson, M. G., Pencina, M. J., Hamburg, N. M., . . Benjamin, E. J. (2010). Arterial stiffness and cardiovascular events: the Framingham Heart Study. Circulation, 121(4), 505-511. doi: CIRCULATIONAHA.109.886655 [pii]10.1161/CIRCULATIONAHA.109.886655

Naidu, M. U., Reddy, B. M., Yashmaina, S., Patnaik, A. N., \& Rani, P. U. (2005). Validity and reproducibility of arterial pulse wave velocity measurement using new device with oscillometric technique: a pilot study. Biomed Eng Online, 4, 49. doi: 10.1186/1475-925X-4-49

Narimatsu, K., Takatani, S., \& Ohmori, K. (2001). A multi-element carotid tonometry sensor for non-invasive measurement of pulse wave velocity. Front Med Biol Eng, 11(1), 45-58.

O'Rourke, M., Farnsworth, A., \& O'Rourke, J. (2008). Aortic dimensions and stiffness in normal adults. JACC Cardiovasc Imaging, 1(6), 749-751. doi: 10.1016/j.jcmg.2008.08.002

O'Rourke, M. F., \& Adji, A. (2012). Noninvasive studies of central aortic pressure. Curr Hypertens Rep, 14(1), 8-20. doi: $10.1007 / \mathrm{s} 11906-011-0236-5$

O'Rourke, M. F., \& Hashimoto, J. (2008). Arterial stiffness: a modifiable cardiovascular risk factor? J Cardiopulm Rehabil Prev, 28(4), 225-237. doi: 10.1097/01.HCR.0000327179.21498.3801273116-200807000-00001 [pii]

O'Rourke, M. F., Safar, M. E., \& Roman, M. J. (2010). Letter by O'Rourke et al regarding article, "Arterial stiffness and cardiovascular events: the Framingham Heart Study". Circulation, 122(19), e512; author reply e515. doi: 122/19/e512 [pii]10.1161/CIRCULATIONAHA.110.945089

Palatini, P., Casiglia, E., Gasowski, J., Gluszek, J., Jankowski, P., Narkiewicz, K., . . Kawecka-Jaszcz, K. (2011). Arterial stiffness, central hemodynamics, and cardiovascular risk in hypertension. Vasc Health Risk Manag, 7, 725-739. doi: 10.2147/VHRM.S25270

Rogers, W. J., Hu, Y. L., Coast, D., Vido, D. A., Kramer, C. M., Pyeritz, R. E., \& Reichek, N. (2001). Age-associated changes in regional aortic pulse wave velocity. J Am Coll Cardiol, 38(4), 1123-1129.

Safar, M., \& O'Rourke M, F. (2006). Handbook of Hypertension: Arterial Stiffness in Hypertension. (Vol. 23). London: Elsevier.

Salvi, P., \& SpringerLink (Online service). (2012). Pulse waves how vascular hemodynamics affects blood pressure (pp. 1 online resource (xii, 138 p.) ill.). Retrieved from http://dx.doi.org/10.1007/978-88-470-2439-7

Vermeersch, S. J., Rietzschel, E. R., De Buyzere, M. L., De Bacquer, D., De Backer, G., Van Bortel, L. M., . . Segers, P. (2008). Determining carotid artery pressure from scaled diameter waveforms: comparison and validation of calibration techniques in 2026 subjects. Physiol Meas, 29(11), 1267-1280. doi: 10.1088/0967-3334/29/11/003Aikenhead, G. S., (1996). Science education: Border crossing into the subculture of science. Studies in Science Education, 27, 1-52. 